\title{
Mucosal leishmaniasis: urgent need for more research
}

\author{
Edgar Marcelino Carvalho ${ }^{[1]}$, Alejandro Llanos-Cuentas ${ }^{[2]}$ \\ and Gustavo Adolfo Sierra Romero ${ }^{[3]}$
}

\begin{abstract}
[1]. Serviço de Imunologia, Centro de Pesquisa Gonçalo Moniz, Fundação Oswaldo Cruz, Salvador, BA, Brasil. [2]. Instituto de Medicina Tropical Alexander von Humboldt, Universidad Peruana Cayetano Heredia, Lima, Peru. [3]. Núcleo de Medicina Tropical, Faculdade de Medicina, Universidade de Brasília, Brasília, DF, Brasil.
\end{abstract}

Leishmaniasis is one of the most neglected tropical diseases and is strongly associated with poverty ${ }^{1}$. Mucocutaneous or mucosal leishmaniasis (ML) is an important and devastating form of the disease that usually develops after the primary cutaneous lesion or as a primary manifestation. ML pathogenesis is related to parasite and host factors, in particular, the host's immunological response ${ }^{2}$. ML may present many distinct manifestations, from lesions limited to the nasal and oral cavity (mild stage), involvement of the epiglottis (moderate stage), to the involvement of the vocal cords, subglottic region, trachea, and even bronchi (severe stage). In some cases, mucosal involvement may lead to the destruction of the face structure, and respiratory failure associated with death ${ }^{2,3}$. The disfigurement related to ML often leads to economic losses, severe stigmatization, and may cause psychological disorders as well as restrict social participation of the individual ${ }^{4}$. Over $90 \%$ of ML cases occur in Bolivia, Brazil, Ethiopia, and Peru. In the American region, ML represents around $4 \%$ of tegumentary leishmaniasis cases ${ }^{5}$; however, in some countries this proportion can be even higher. In 2013, Paraguay and Argentina reported $37 \%$ and $14.4 \%$ of ML cases, respectively ${ }^{6}$. In 2014, 1,953 ML cases were reported in the American region, with 1,016 in Brazil alone 5 .

Current treatment recommendations for ML are: 1) pentavalent antimonials $\left.\left(\mathrm{Sb}^{v}\right) ; 2\right) \mathrm{Sb}^{v}$ in combination with oral pentoxifylline, as a first line treatment; 3) liposomal amphotericin B (L-AmB) in case of therapeutic failure or special situations; 4) amphotericin $\mathrm{B}$ deoxycholate (AB); 5) pentamidine isethionate (PT); or 6) miltefosine (MF) ${ }^{7}$. All of these drugs have limitations in terms of toxicity, variable efficacy, and inconvenient treatment schedules (e.g. long administration times and parenteral administration). Moreover, the treatment outcome also depends on the stage of the disease.

Corresponding author: Dr. Edgar Marcelino Carvalho.

e-mail: edgar@ufba.br; imuno@ufba.br

Received 29 November 2017

Accepted 2 January 2018
Higher cure rates are obtained when the lesions are limited to the nose and mouth (mild manifestation).

Cure rates of $\mathrm{Sb}^{v}$ range from $30-100 \%$, depending on the geographical area and the severity of the disease ${ }^{8}$. It has been shown in a series of patients in Peru that the cure rate was $40.9 \%$ in the moderate stage and $7.1 \%$ in the severe stage ${ }^{3}$ indicating that $\mathrm{Sb}^{v}$ should not be recommended as a therapeutic option for these cases. Given the variable cure rates reported with antimonial drugs and its well-known toxicity, its use for the treatment of ML is limited.

Although the quality of the evidence is weak, the combination of oral pentoxifylline and $\mathrm{Sb}^{v}$ reduced the relapse rate and accelerated cure compared to $\mathrm{Sb}^{v}$ alone ${ }^{8}$. A recent study of 205 patients with ML in Lima, Peru has shown an increase in the cure rate of $61 \%$ in the group treated with $\mathrm{Sb}^{v}$ alone versus $79 \%(\mathrm{p}=0.011)$ in the group treated with $\mathrm{Sb}^{\mathrm{v}}$ plus pentoxifylline $\mathrm{s}^{5}$.

Amphotericin B deoxycholate (very low quality of evidence) is effective in more than $90 \%$ of patients and it also cures cases refractory to $\mathrm{Sb}^{\mathrm{v} 2}$. However, the total required dose has not yet been established. In Brazil and Peru, most patients achieved cure with a cumulative dose of $25 \mathrm{mg}$ (approximately 42 doses). In addition, the toxicity of $\mathrm{AB}$, mainly related to kidney failure, is a limiting factor for its use.

Limited data are available on the use of PT. It showed excellent results in 27 patients with moderate to high grade lesions in Brazil ${ }^{9}$. Nevertheless, the quality of evidence is very low, and its use is limited by its toxicity, mainly pancreatic, that requires careful monitoring.

The only oral option, MF was shown to be efficacious in one randomized clinical trial conducted in Bolivia ${ }^{10}$. However, therapeutic failure was observed in $17 \%$ of patients with a mild manifestation of the disease and $42 \%$ with a severe stage, characterized by the involvement of the palate, pharynx, and larynx ${ }^{11}$. Despite the small number of patients, the Peruvian study was not very positive, with only one out of 10 patients cured $^{3}$. In addition, MF may cause gastrointestinal side-effects and its teratogenicity is a limiting factor for women.

Some studies using L-AmB have been published; however, these studies were not controlled and included a limited number 
of patients, hence it is difficult to draw any conclusions regarding the most effective dose/regimen. According to the World Health Organization, a dose of $2-3 \mathrm{mg} / \mathrm{kg}$ for at least 20 days provides a cure rate of $80-90 \% \%^{8}$. Three published case series conducted in Brazil, including a total of 15 patients with ML caused by Leishmania braziliensis who received L-AmB 2-3mg/ $\mathrm{kg} /$ day until a total dose of $40-50 \mathrm{mg} / \mathrm{kg}$, achieved response rates of $83.3-100 \%{ }^{12}$. In a retrospective study conducted in Brazil, a $93.1 \%$ cure rate was achieved in 29 patients who received dose ranges of L-AmB of $18.2-55.2 \mathrm{mg} / \mathrm{kg}^{2}$. A recent unpublished clinical trial in Peru showed a cure rate of $90 \%$ in severe cases treated with $3 \mathrm{mg} / \mathrm{kg} /$ day for 21 days $^{3}$.

Current efforts to identify new treatments for leishmaniasis are largely focused on visceral leishmaniasis and to a lesser extent on cutaneous leishmaniasis; there are currently no efforts to identify new treatment options for patients with ML, despite the difficulties and limitations of using current treatment options and the severe complications of the disease, which include stigma, mutilation, and even death.

Even though progress has been made in the identification and preclinical development of compounds against Leishmania species, in a short period of time (5-7 years), currently available treatments will probably represent almost the entire therapeutic arsenal for the coming years. Hence, there is a great need to explore ways to optimize the use of existing tools and generate solid evidence in order to improve the treatment of ML.

Acknowledging these constraints, the $4^{\text {th }}$ Leishmaniasis Research Network (redeLEISH) meeting proposed a special session on Mucosal Leishmaniasis which took place during the WorldLeish-6 Congress in Toledo on May 16, 2017.

The investigators present at the meeting agreed that there is an urgent need for:

- More incentives and funding to conduct well-structured clinical trials with L-AmB or other drugs and combinations of treatments in order to develop more effective and safer treatments for ML and to generate robust evidence for the adoption of new treatment recommendations.

- Encouraging investment to develop new rapid and noninvasive diagnostic tests.

- Raising awareness of the social problem of ML and engaging governments to support scientific research related to public health and ML.

- The promotion of better access to early diagnostic tools and treatment for patients with ML.
Thus, this proposal was launched during the ML session at the $4^{\text {th }}$ meeting of redeLEISH at the WorldLeish- 6 Congress and approved by the scientific community and social movements at the $53^{\text {rd }}$ meeting of the Brazilian Society of Tropical Medicine. More people have embraced the cause and today we count 137 signatures. Virtual signatures are collected at the following link: https://www.abaixoassinado.org/abaixoassinados/37330.

In view of the current situation and the limited options available to treat ML, the participants of the meeting expressed their support to increase efforts to improve treatment of ML in line with the above statement.

\section{Conflict of interest}

The authors declare that they have no conflicts of interest.

\section{REFERENCES}

1. Alvar J, Yactayo S, Bern C. Leishmaniasis and poverty. Trends in Parasitol. 2006;22(12):552-6.

2. Carvalho E, Machado P. The importance of seeking therapeutic solutions for mucosal leishmaniasis in Latin America. Special communication for InfoLeish $\mathrm{n}^{\circ} 2,2017$.

3. Llanos-Cuentas A. Clinical and therapeutic update on mucosal leishmaniasis. Special communication for InfoLeish no 2, 2017.

4. Alonso LM, Alvar J. Stigmatizing neglected tropical diseases: a systematic review. Social Medicine. 2010;5(4):218-27.

5. Pan American Health Organization. Leishmaniases epidemiological report in Americas. 2016.

6. Pan American Health Organization. Leishmaniases epidemiological report in Americas, 2015

7. Pan American Health Organization. (PAHO). Recommendations for the treatment of leishmaniasis in the Americas. Washington, DC: PAHO; 2013.

8. World Health Organization. (WHO). Control of the leishmaniases, WHO Technical Report Series, 949. Geneva: WHO; 2010.

9. Amato VS, Tuon FF, Siqueira AM, Nicodemo AC, Amato Neto V. Treatment of mucosal leishmaniasis in Latin America: systematic review. Am J Trop Med Hyg. 2007;77(2):266-74.

10. Soto J, Rea J, Valderrama M, Toledo J, Valda L, Ardiles J, et al. Efficacy of extended (six weeks) treatment with miltefosine for mucosal leishmaniasis in Bolivia. Am J Med Hyg. 2009;81(3):387-9.

11. Soto J, Toledo J, Valda L, Balderrama M, Rea I, Parra R, et al. Treatment of Bolivian mucosal leishmaniasis with miltefosine. Clin Infect Dis. 2007;44(3):350-6.

12. Monge-Mailo B, López-Vélez R. Therapeutic options for old world cutaneous leishmaniasis and new world cutaneous and mucocutaneous leishmaniasis. Drugs. 2013;73(17):1889-920. 